\title{
Hobbing Strategy and Performance Analyses of Linkage Models for Non-Circular Helical Gears Based on Four-Axis Linkage
}

\author{
Youyu Liu1,2,* - Jiang Han ${ }^{2}-$ Lian Xia $^{2}$ - Xiaoqing Tian ${ }^{2}$ \\ ${ }^{1}$ School of Mechanical and Automotive Engineering, Anhui Polytechnic University, China \\ 2 Institute of CIMS, Hefei University of Technology, China
}

\begin{abstract}
The application of non-circular helical gears has been largely restricted to their manufacturing techniques. For realization of their hobbing, a kind of hobbing strategy based on a four-axis linkage and some fundamental hobbing models have been built by a generating method of helical tooling rack. This method includes profile-linkage models and extra rotation models. Based on the strategy and the models achieved, eighteen kinds of schemes and functional models have been developed according to the variety of hobbing processes in profile movement, axial movement, and extra rotation, which have an effect on profile precision, axial precision and control performance. The effect is analyzed from the aspects of profile-hobbing performance, axial movement performance and extra rotation performance, by using a 3D machining simulation. Excellent strategy and models have been singled out progressively. Finally, the excellent strategy and model (constant revolving velocity of hob \& constant axial speed for workpiece \& extra rotation of workpiece) is obtained with the characteristics of high precision, high efficiency and ease of control. The excellent strategy and model have been demonstrated to be valid by hobbing tests. The results between the test for tooth flanks and the computer simulations are in good agreement. This work provides the options of schemes for the manufacture of non-circular helical gears, and will promote its application significantly.
\end{abstract}

Keywords: non-circular helical gears, four-axis linkage, hobbing, linkage models, machining simulation

\section{INTRODUCTION}

Non-circular gears synthesize the advantages of circular gears and cam mechanisms, and can deliver a combination of high output power and excellent level accuracy with a continuously variable transmission. Non-circular gears have been applied to construction machineries, machine tools, automotives, aerospaces and other fields [1] to [3]. Most of current research is focused on non-circular spur gears. However, the studies for the design and manufacture of non-circular helical gears are few. For the advantages such as larger contact ratio, less impact and noise, smaller teeth be free of undercut, non-circular gears have a fairly good application prospect.

Recently, remarkable progress of non-circular gears has been made in the research of implementing their function and analyzing their performance [4] and [5]. As for their manufacturing technology, some academics have conducted some research. $\mathrm{Wu}$ et al. [6] have proposed a numerical algorithm of the tooth profile of non-circular gears based on the characteristics of cutter envelope, which can only be used for non-circular spur gears by wire electric discharge machining. In the last few years, new approaches to gear cutting have emerged. For example, José Luís Huertas Talón et al. [7] have offered a method of manufacturing a spur gear in Ti-6Al-4V alloy using a wire electrical discharge machine. In addition, a precision gear was cut on 0.4 $\mathrm{mm}$ stainless steel sheets with fiber laser precision processing system in paper [8]. The above-mentioned methods can also be applied to non-circular spur gears regardless of their shape of pitch curve, but does not apply at all to non-circular helical gears. Moreover, those methods above, being hopelessly inefficient, are suitable only for such gears with special materials or extreme thinness. Hobbing is still our first choice for its high efficiency. Hou and Liu [9] have researched a meshing theory model for the manufacturing of noncircular helical gears by a generating method of helical tooling rack, and analysed the geometric features of the tooth profile, but provided no processing program for them.

In this paper, some available hobbing schemes and functional models have been developed based on the strategy of the four-axis linkage and the fundamental linkage models. Profile precision, axial precision and control performance have been analyzed according to the variety of the hobbing process in profile movement, axial movement, and extra rotation by using 3D machining simulation. Thus, excellent strategy and models have been singled out progressively.

\section{HOBBING MODEL FOR NON-CIRCULAR HELICAL GEARS BASED ON A FOUR-AXIS LINKAGE}

\subsection{Hobbing Strategy Based on a Four-Axis Linkage}

As shown in Fig. 1, the hob rotation $\omega_{b}$ and the workpiece rotation $\omega_{c}$ must keep a strict transmission ratio, which generates meshing movement. Meanwhile, the workpiece must move along the $y$-axis 
(namely $\boldsymbol{v}_{y}$ ) to form non-circular gears pitch curve. Moreover, the hob should move along the $z$-axis, namely $\boldsymbol{v}_{z}$, to cut a full-depth tooth. For helical gears, extra rotation is essential, which can be implemented by extra rotation of workpiece, namely $\Delta \omega_{c}$, or that of hob, namely $\Delta \omega_{b}$. All of the above is the basic hobbing strategy based on a four-axis linkage.

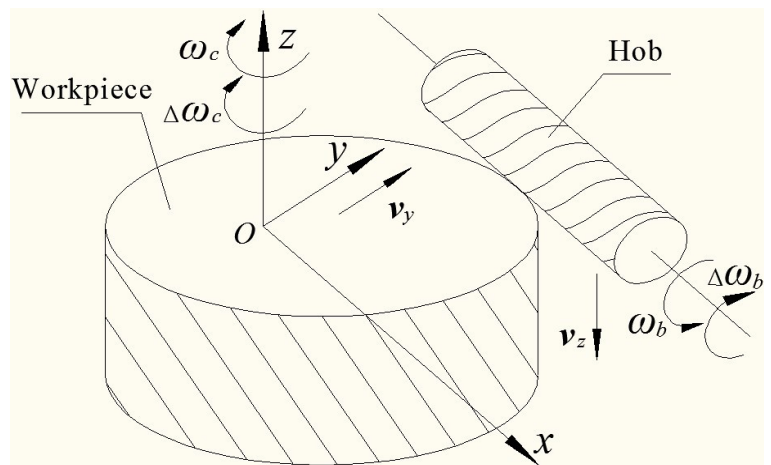

Fig. 1. Schematic diagram of a four-axis hobbing process

Compared to the four-axis linkage, the workpiece should move along the $x$-axis (namely $\boldsymbol{v}_{x}$ ) based on the five-axes linkage, which would increase the number of linkage axes. In addition, machining accuracy may be decreased due to a clearance while reciprocating movement [10]. Consequently, Four-axis Linkage method is a good hobbing strategy for non-circular helical gears.

\subsection{Fundamental Hobbing Models Based on a Four-Axis Linkage}

According to the meshing theory of hobbing noncircular helical gears by a helical tooling rack [9], the pitch line of the helical tooling rack rolls along the pitch curve of the non-circular helical gears without slip. A revolving hob and an extra rotation $\left(\Delta \omega_{c}\right.$ or $\left.\Delta \omega_{b}\right)$ can realize the functions of the helical tooling rack. The cross-section of workpiece is shown in Fig. 2. The tooling rack is formed by the hob projected to the end-face, the pitch line of which tangents to the point $P$ with the pitch curve of the non-circular helical gears on the end-face. For pure rolling between them, the motion of the workpiece is $s$, and that of the tooling rack is $l$.

As shown in Fig. 2, $S(o x y z)$ is a machine coordinate system, the $x$-axis and $z$-axis of which are independently parallel to the pitch line of the hob and the turning spindle axis of the workpiece, and the $y$-axis of which passes through the turning spindle axis of that. $S_{b}\left(o_{b} x_{b} y_{b} z_{b}\right)$ is a cutting tool coordinate system, moved with the helical tooling rack, and each axis of which is parallel with that of $S($ oxyz). At the beginning, the $S_{b}\left(o_{b} x_{b} y_{b} z_{b}\right)$ and the $S($ oxyz) coodinate systems are kept in superposition. $S_{c}\left(o_{c} x_{c} y_{c} z_{c}\right)$ is a workpiece coordinate system, it revolves with the workpiece, and the $z_{c}$-axis of which is coincident with the turning spindle axis of the workpiece. At the beginning, the $x_{c}$-axis is parallel with the $y$-axis, and the $y_{c}$-axis is parallel with the $x$-axis. Moreover, we build a polar coordinate system that has a pole $o_{c}$ and polar axis $x_{c}$. The pitch curve equation of the noncircular helical gears is $r=r(\theta)$. The modulus of the polar radius is $r$; the polar angle is $\theta$; the polar-angular velocity is $\omega$. The forward direction of the $\theta$, the $\varphi_{c}$, and the $\omega_{c}$ is as shown in Fig. 2.

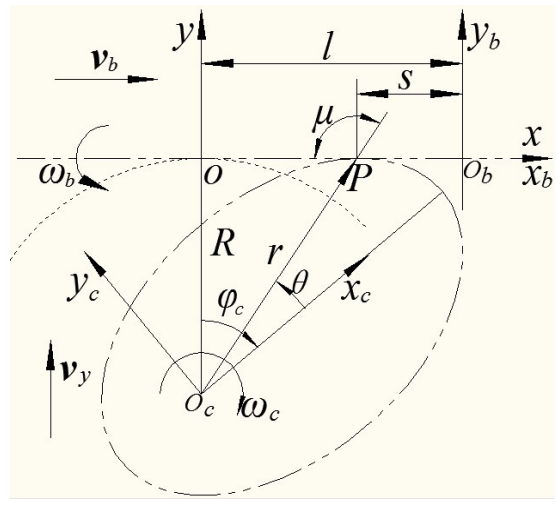

Fig. 2. Cross-section of workpiece

\subsubsection{Profile-Linkage Models}

As shown in Fig. 2, the angle between the polar radius and the tangent of the pitch curve of the workpiece is $\mu$. From the theory of calculus [11], we can infer the following.

$$
\begin{gathered}
\mu=\arctan (r / d r / d \theta) \quad(0 \leq \mu<\pi), \\
\sin \mu=r / \sqrt{r^{2}+(d r / d \theta)^{2}},
\end{gathered}
$$

then,

$$
\frac{d \mu}{d t}=\frac{d \mu}{d \theta} \cdot \frac{d \theta}{d t}=\frac{(d r / d \theta)^{2}-r \cdot d^{2} r / d \theta^{2}}{r^{2}+(d r / d \theta)^{2}} \cdot \frac{d \theta}{d t} .
$$

From Fig. 2,

$$
\theta=\varphi_{c}-\mu+\pi / 2,
$$

then,

$$
\frac{d \theta}{d t}=\frac{d \varphi_{c}}{d t}-\frac{d \mu}{d t}=\omega_{c}-\frac{d \mu}{d t} .
$$

The Eq. (2) is substituted in the Eq. (4), then 


$$
\omega_{c}=\frac{r^{2}+2(d r / d \theta)^{2}-r \cdot d^{2} r / d \theta^{2}}{r^{2}+(d r / d \theta)^{2}} \cdot \frac{d \theta}{d t} .
$$

For the pitch curve $(r=r(\theta))$ being a kind of non-circular curve, $\omega_{c}$ varies nonlinearly with $\omega_{b}$ in hobbing. As shown in Fig. 2, there is an equialent helical gear that has center of rotation, $o_{c}$, and reference radius corresponding to the distance $R$, which is from $o_{c}$ to the tangent of the pitch curve. Some parameters such as the helix angle $\beta_{c}$, the normal module $m_{n}$, and the transverse module $m_{t}$ are the same as those of non-circular helical gears. The equivalent teeth number is marked as $Z_{v}$, then,

$$
\frac{\omega_{b}}{\omega_{c}}=\frac{Z_{v}}{K}=\frac{2 R}{K m_{t}}=\frac{2 R \cos \beta_{c}}{K m_{n}},
$$

where the $K$ is the lobes of hob.

$$
R=r \sin (\pi-\mu)=r \sin \mu .
$$

The Eqs. (1), (5) and (7) are substituted in the Eq. (6), then,

$$
\omega_{b}=\frac{2 r^{2} \cos \beta_{c}\left(r^{2}+2(d r / d \theta)^{2}-r d^{2} r / d \theta^{2}\right)}{K m_{n}\left(r^{2}+(d r / d \theta)^{2}\right)^{3 / 2}} \cdot \frac{d \theta}{d t} .
$$

From Eq. (7), the velocity of the workpiece along the normal direction of the pitch line of the tooling rack can be deduced.

$v_{y}=\frac{d R}{d t}=\frac{r d r / d \theta\left[r^{2}+2(d r / d \theta)^{2}-r d^{2} r / d \theta^{2}\right]}{\left(r^{2}+(d r / d \theta)^{2}\right)^{3 / 2}} \cdot \frac{d \theta}{d t}$.

From Eqs. (5), (8) and (9), a fundamental linkage model based on hobbing is elicited as follows.

$$
\begin{gathered}
v_{y}=r(d r / d \theta) \omega_{c} / \sqrt{r^{2}+(d r / d \theta)^{2}}, \\
\omega_{c}=K m_{n} \sqrt{r^{2}+(d r / d \theta)^{2}} \omega_{b} /\left(2 r^{2} \cos \beta_{c}\right) .
\end{gathered}
$$

\subsubsection{Extra Rotation Models}

As mentioned previously, extra rotation can be implemented by a workpiece or hob.

\subsubsection{Extra Rotation of Workpiece}

If an extra rotation $\Delta \omega_{c}$ is added to the workpiece rotation $\omega_{c}$, marked as "A", the workpiece should rotate a week additionally while moving a screw lead along the axle of that [12].

$$
\int_{0}^{t} r \Delta \omega_{c} d t=\tan \beta_{c} \int_{0}^{t} v_{z} d t
$$

Differentiating Eq. (11) and simplifying it:

$$
\Delta \omega_{c}=\left(v_{z} \tan \beta_{c}\right) / r .
$$

The resultant angular velocity $\omega_{c}^{*}$ is as follows.

$$
\omega_{c}^{*}=\omega_{c} \pm \Delta \omega_{c},
$$

where the "+" is adopted while the direction of the helix of the hob is in accordance with that of the gears; otherwise, the "-" is adopted.

\subsubsection{Extra Rotation of Hob}

As shown in Fig. 3, an extra rotation $\Delta \omega_{b}$ is added to the hob rotation $\omega_{b}$, marked as " $\mathrm{B}$ ". The line $t-t$ is a tangent to the inclined tooth flank in the point of $P$. The component of the vertical motion $v_{z}$ related to the common normal line $n-n$ is $\boldsymbol{v}_{z}^{n} \cdot \boldsymbol{v}_{\Delta}$ represents an extra linear velocity in the point of $P$ due to $\Delta \omega_{b}$, the component of which related to the common normal line is $\boldsymbol{v}_{\Delta}^{n}$.

$$
\begin{aligned}
& v_{z}^{n}=v_{z} \sin \beta_{c}, \\
& v_{\Delta}=K m_{t} \Delta \omega_{b} / 2=K m_{n} \Delta \omega_{b} /\left(2 \sin \lambda_{b}\right), \\
& v_{\Delta}^{n}=v_{\Delta} \sin \lambda_{b},
\end{aligned}
$$

where the " $\lambda_{b}$ " is the lead ascending angle of the hob.

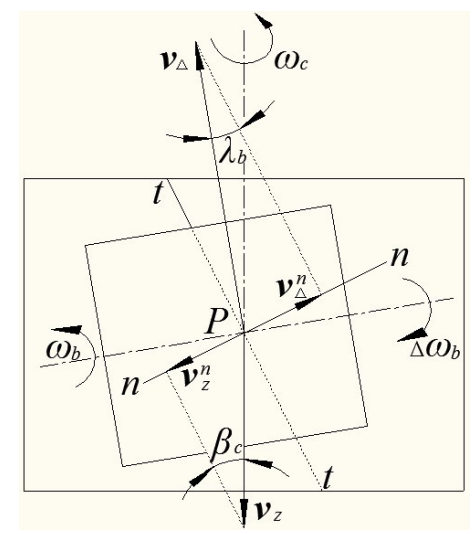

Fig. 3. Common tangent plane of hobbing

The normal velocity of the hob in the meshing point should equal to that of the workpiece [10].

$$
v_{z}^{n}=v_{\Delta}^{n} .
$$


From Eqs. (14) and (15),

$$
\Delta \omega_{b}=\left(2 v_{z} \sin \beta_{c}\right) / K m_{n},
$$

the resultant angular velocity $\omega_{b}^{*}$ is as follows:

$$
\omega_{b}^{*}=\omega_{b} \mp \Delta \omega_{b},
$$

where the "-" is adopted while the direction of the helix of the hob is in accordance with that of the gears; otherwise, the "+" is adopted.

\section{REALIZATION STRATEGY OF THE LINKAGE MODEL}

There are three kinds of hobbing methods [9], such as constant revolving velocity of hob (marked as "U"), constant revolving velocity of workpiece (marked as "V") and constant polar-angular velocity of workpiece (marked as "W"), in the cross-section of non-circular gears. The constant revolving velocity of hob implies that the velocity of hob keeps constant, consequently the workpiece rotates at constant arc length at the same time. The constant revolving velocity of workpiece implies that the workpiece rotates with a constant angular velocity. The constant polar-angular velocity of the workpiece implies that the workpiece rotates with constant polar-angular velocity. There are three kinds of movement methods for the axial movement $\boldsymbol{v}_{z}$, such as being coupled with $\omega_{b}$ (marked as " $\alpha$ "), being coupled with $\omega_{c}$ (marked as " $\beta$ ") and constant axial speed (marked as " $\gamma$ ").

For the variety of hobbing process in profile movement, axial movement, and extra rotation, there are eighteen $\left(\mathrm{C}_{3}^{1} \mathrm{C}_{3}^{1} \mathrm{C}_{2}^{1}=18\right)$ kinds of schemes and functional models that can be realized. For the constant revolving velocity of hob, some schemes

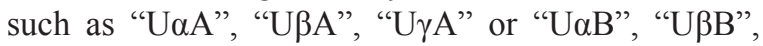
"UүB" can be constructed from Eqs. (1) and (2), or Eqs. (1) and (3), and the mathematical model of which is Eqs. (18) or (19). The $\boldsymbol{v}_{z}=f\left(\omega_{b}, \omega_{c}, k_{3}\right)$ in Eqs. (18) and (19) can be determined based on the linkage methods of the axial movement, as Eq. (20). For the constant revolving velocity of workpiece, some schemes such as "V $\alpha \mathrm{A}$ ", "V $\beta A$ ", "V $\gamma \mathrm{A}$ " or "V $\alpha \mathrm{B}$ ", "V $\beta B$ ", "V $\gamma \mathrm{B}$ " can be constructed from Eqs. (1) and (2), or Eqs. (1) and (3), and the mathematical model of which is Eqs. (21) or (22). For the constant polarangular velocity of the workpiece, some schemes such as "W $\alpha \mathrm{A}$ ", "W $\beta A$ ", "W $\gamma \mathrm{A}$ " or "W $\alpha \mathrm{B}$ ", "W $\beta B$ ", "W $\gamma \mathrm{B}$ " can be constructed from Eqs. (1) and (2), or Eqs. (1) and (3), and the mathematical model of which is Eqs. (23) or (24).

$$
\begin{gathered}
v_{y}=\left[K m_{n}(d r / d \theta) / 2 r \cos \beta_{c}\right] \omega_{b}, \\
\omega_{c}^{*}=\frac{K m_{n} \sqrt{r^{2}+(d r / d \theta)^{2}}}{2 r^{2} \cos \beta_{c}} \omega_{b} \pm \frac{\tan \beta_{c}}{r} v_{z}, \\
v_{z}=f\left(\omega_{b}, \omega_{c}, k_{3}\right),
\end{gathered}
$$

$$
\begin{gathered}
v_{y}=\left[K m_{n}(d r / d \theta) / 2 r \cos \beta_{c}\right] \omega_{b}, \\
\omega_{c}=\left[K m_{n} \sqrt{r^{2}+(d r / d \theta)^{2}} / 2 r^{2} \cos \beta_{c}\right] \omega_{b}, \\
\omega_{b}^{*}=\omega_{b} \mp\left[2 \sin \beta_{c} / K m_{n}\right] v_{z}, \\
v_{z}=f\left(\omega_{b}, \omega_{c}, k_{3}\right),
\end{gathered}
$$$$
v_{z}=f\left(\omega_{b}, \omega_{c}, k_{3}\right)=\left\{\begin{array}{ll}
k_{1} \omega_{b} & (\alpha) \\
k_{2} \omega_{c} & (\beta) \\
k_{3} & (\gamma)
\end{array},\right.
$$

$$
\begin{gathered}
v_{y}=\frac{r(d r / d \theta)\left[r^{2}+2(d r / d \theta)^{2}-r\left(d^{2} r / d \theta^{2}\right)\right]}{\left[r^{2}+(d r / d \theta)^{2}\right]^{3 / 2}} \omega, \\
\omega_{b}=\frac{2 r^{2} \cos \beta_{c}\left[r^{2}+2(d r / d \theta)^{2}-r\left(d^{2} r / d \theta^{2}\right)\right]}{K m_{n}\left[r^{2}+(d r / d \theta)^{2}\right]^{3 / 2}} \omega, \\
\omega_{c}^{*}=\frac{r^{2}+2(d r / d \theta)^{2}-r\left(d^{2} r / d \theta^{2}\right)}{r^{2}+(d r / d \theta)^{2}} \omega \pm \frac{v_{z} \tan \beta_{c}}{r}, \\
v_{z}=f\left(\omega_{b}, \omega_{c}, k_{3}\right),
\end{gathered}
$$

$$
\begin{gathered}
v_{y}=\left[r(d r / d \theta) / \sqrt{r^{2}+(d r / d \theta)^{2}}\right] \omega_{c}, \\
\omega_{b}=\frac{2 r^{2} \cos \beta_{c}}{K m_{n} \sqrt{r^{2}+(d r / d \theta)^{2}}} \omega_{c} \mp \frac{2 \sin \beta_{c}}{K m_{n}} v_{z}, \\
v_{z}=f\left(\omega_{b}, \omega_{c}, k_{3}\right),
\end{gathered}
$$




$$
\begin{gathered}
v_{y}=\frac{r(d r / d \theta)\left[r^{2}+2(d r / d \theta)^{2}-r\left(d^{2} r / d \theta^{2}\right)\right]}{\left[r^{2}+(d r / d \theta)^{2}\right]^{3 / 2}} \omega, \\
\omega_{b}^{*}=\frac{2 r^{2} \cos \beta_{c}\left[r^{2}+2(d r / d \theta)^{2}-r\left(d^{2} r / d \theta^{2}\right)\right]}{K m_{n}\left[r^{2}+(d r / d \theta)^{2}\right]^{3 / 2}} \omega \mp \frac{2 \sin \beta_{c}}{K m_{n}} v_{z}, \\
\omega_{c}=\left[r^{2}+2(d r / d \theta)^{2}-r\left(d^{2} r / d \theta^{2}\right)\right] \omega /\left[r^{2}+(d r / d \theta)^{2}\right], \\
v_{z}=f\left(\omega_{b}, \omega_{c}, k_{3}\right),
\end{gathered}
$$

\section{ANALYSIS AND SELECTION OF LINKAGE MODELS BASED ON VIRTUAL MACHINING}

Including the basic function of hobbing and the linkage strategy properly, an excellent linkage model should have the performance qualities of high precision, high efficiency and ease of control. An elliptical helical gear will be analyzed as an example in this paper, which has the essential feature of any non-circular helical gears. The conclusions drawn can be expanded to other non-circular helical gears with arbitrary pitch curve. Its principal parameters are as follows: major semi-axis $a=100 \mathrm{~mm}$, eccentricity $e=0.6, K=1, m_{n}=5 \mathrm{~mm}, \beta_{c}=14.251^{\circ}$, number of teeth $Z=35$, tooth width $b=50 \mathrm{~mm}$. The mathematical models (see Eqs. (18) to (24)) applied to the elliptical helical gear are discussed from three aspects. These are profile precision, axial precision and control performance by virtual machining process using MATLAB [13], to single out the excellent strategy and models progressively. For example, three kinds of models of constant axial speed $(\gamma)$ \& extra rotation of the workpiece (" $A$ ") are simulated and shown in Fig. 4. a)

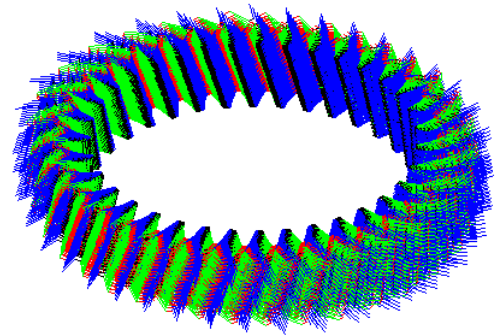

b)

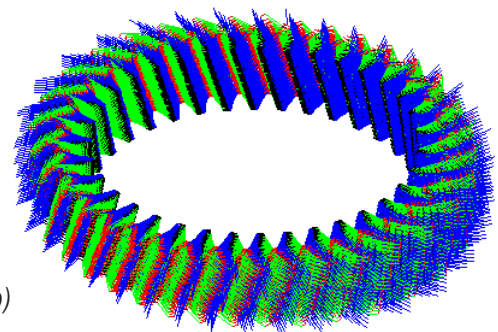

c)

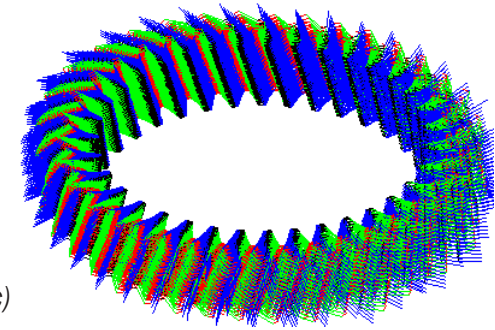

Fig. 4. Constant axial speed \& extra rotation of workpiece; a) scheme of the "UYA", b) scheme of the "VYA", c) scheme of the "WYA"

a)
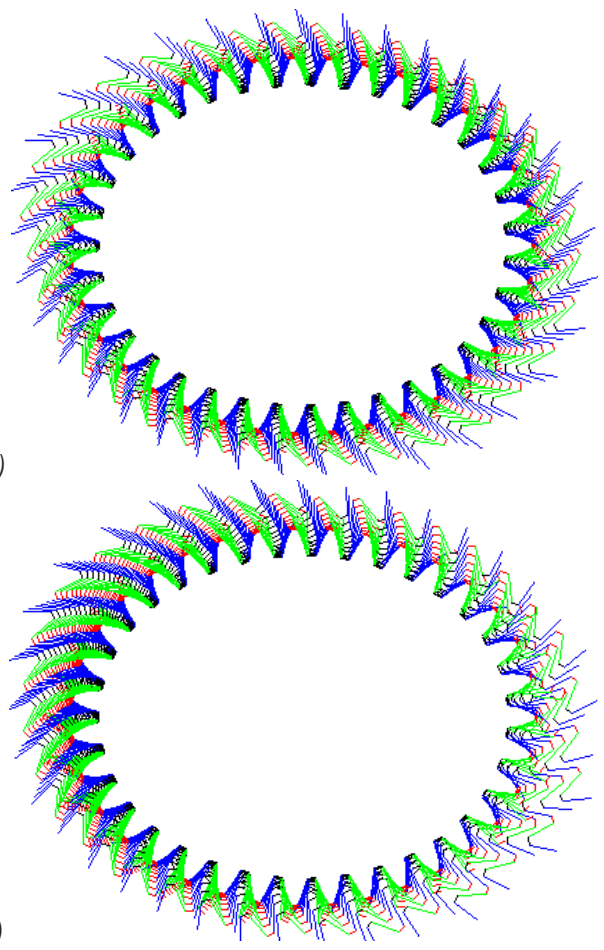

b)
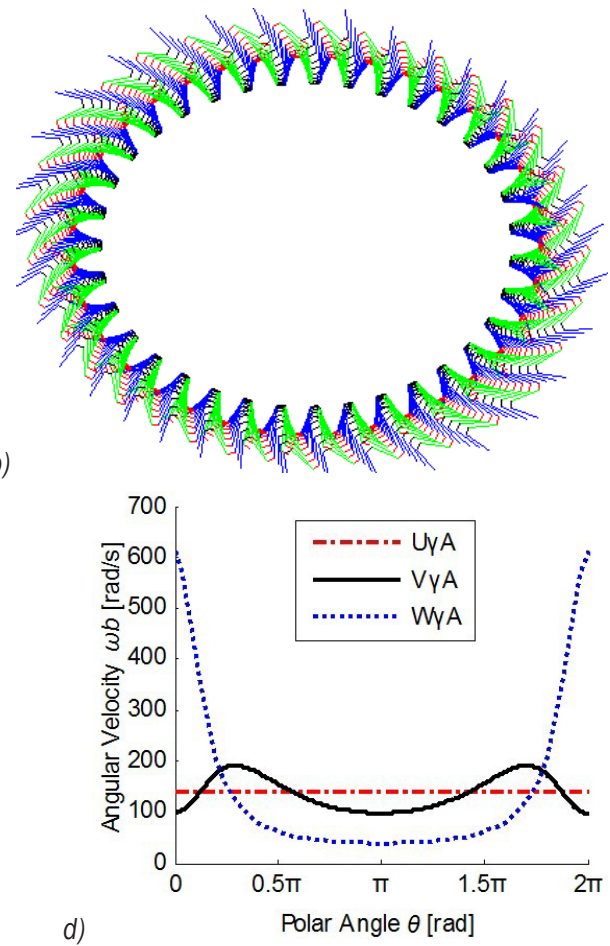

Fig. 5. Profile-hobbing performance; a) scheme of the "UYA", b) scheme of the "VYA", c) scheme of the "WYA", d) graph of $\omega_{b}-\theta$ 


\subsection{Analysis of Profile-Hobbing Performance}

Involute profiles are formed by the meshing movement between $\omega_{b}$ and $\omega_{c}$ on the end-face, which means that the profile precision is determined only by the methods of the profile hobbing process. That is to say, the profile precision has nothing to do with the methods of axial movement and extra rotation. To compare different profile hobbing process features, 3D machining simulations, taking the same axial movement $(\gamma)$ and the same extra rotation ("A") as a case, are conducted and shown in Fig. 4. Their tool paths on the end-face $(z=0)$ are shown in Fig. 5 .

From Figs. 5a and d, we can find that $\omega_{b}$ is constant on any polar angle to the scheme of the "UyA", which makes cutting marks of any tooth uniform, and the precision is basically identical. As illustrated in Figs. $5 \mathrm{a}$ and $\mathrm{d}, \omega_{b}$ of the scheme of the "V $\gamma \mathrm{A}$ " fluctuates. All variables in MATLAB can be inquired conveniently [14]. We find that the minimum $\omega_{b}$ is $98 \mathrm{rad} / \mathrm{s}$ when $\theta$ is 0 or $\pi$, and its maximum is $191.4 \mathrm{rad} / \mathrm{s}$ while $\theta$ is $0.3 \pi$ or $1.7 \pi$. The maximum is 1.95 times as much as the minimum, which makes the cutting marks of every tooth nonuniform. Thus, the precision is different. As shown in Fig. $5 \mathrm{c} d$, the $\omega_{b}$ of the scheme of the "W $\gamma \mathrm{A}$ " fluctuates sharply. The figures show that the maximum $\omega_{b}$ is $612.5 \mathrm{rad} / \mathrm{s}$ when $\theta$ is 0 , and its minimum is $38.28 \mathrm{rad} / \mathrm{s}$ while $\theta$ is $\pi$. The maximum is 16 times more than the minimum, which makes the precision of every tooth vary greatly. The precision of the gear should be measured at its lowest level. Therefore, the constant revolving velocity of hob should be adopted, and can obtain the highest manufacturing precision under a given efficiency. The next ordinal is the constant revolving velocity of the workpiece, the constant pola-angular velocity of the workpiece.

\subsection{Analysis of Axial Movement Performance}

The full-depth tooth can be hobbed through the $v_{z}$ movement, and the axial precision is determined only by the methods of axial movement. To compare the features of different axial movement, three kinds of axial movement methods with the same profile hobbing process ("U") and the same extra rotation ("A") are analyzed. As shown in Fig. 6a, $S_{z}$ is an axial displacement of hob at a polar angle, and $\Delta S_{z}$ is its successive clearance. The $S_{z}$ of the schemes of

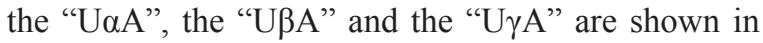
Fig. 6b, which shows that the $S_{z}$ of the "UaA" and the "U $\gamma \mathrm{A}$ " change linearly with $\theta$, but the "U $\beta A$ " varies nonlinearly. The maximum and the minimum each lie in $0.14 \pi$ and $1.86 \pi$. Table 1 lists the final axial displacement of hob and its successive clearance in $0,0.14 \pi, \pi$, and $1.86 \pi$. The successive clearances of

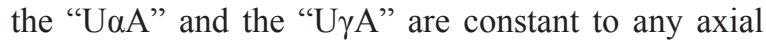
displacement and any polar angle. The successive clearances of the "UßA" are constant to any axial displacement and the same polar angle, not to any polar angle. The minimum of that lies in $0.14 \pi$, which means that the cutting marks are the most plentiful, so the precision is the highest. However, the maximum lies in $1.86 \pi$, which means that the cutting marks are the thinnest, so the precision is the lowest. Therefore,

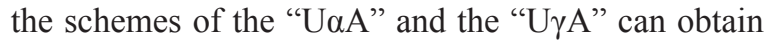
the highest precision under a given efficiency.
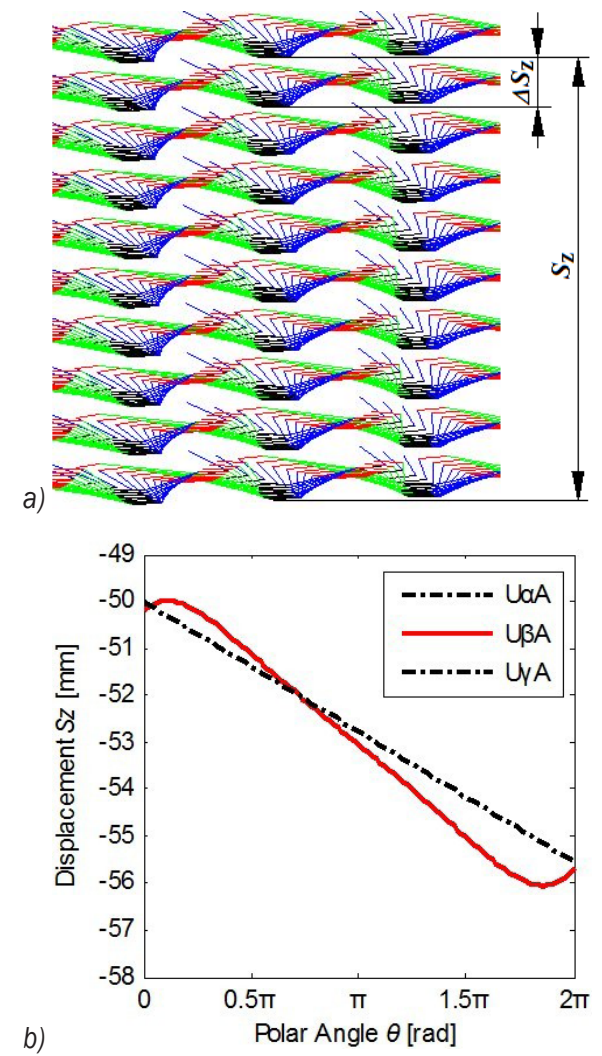

Fig. 6. Axial movement performance;

a) elevation view of tool-path, b) axial displacement

Table 1. Axial displacement and successive clearance in specific polar angle

\begin{tabular}{|c|c|c|c|c|c|}
\hline & $\theta$ [rad] & 0 & $0.14 \pi$ & $\pi$ & $1.86 \pi$ \\
\hline \multirow{2}{*}{$\mathrm{U} \alpha \mathrm{A}$} & $S_{Z}[\mathrm{~mm}]$ & -50.000 & -50.336 & -52.723 & -55.169 \\
\hline & $\Delta S_{Z}[\mathrm{~mm}]$ & -5.000 & -5.000 & -5.000 & -5.000 \\
\hline \multirow{2}{*}{$U \beta A$} & $S_{Z}[\mathrm{~mm}]$ & -50.205 & -50.000 & -52.989 & -56.058 \\
\hline & $\Delta S_{Z}[\mathrm{~mm}]$ & -5.021 & -4.966 & -5.027 & -5.089 \\
\hline \multirow{2}{*}{$U_{\gamma} \mathrm{A}$} & $S_{Z}[\mathrm{~mm}]$ & -50.000 & -50.336 & -52.723 & -55.169 \\
\hline & $\Delta S_{Z}[\mathrm{~mm}]$ & -5.000 & -5.000 & -5.000 & -5.000 \\
\hline
\end{tabular}


The scheme of the "UaA" has the characteristics of constant revolving velocity of hob ("U") and being coupled with $\omega_{b}(\alpha)$. From Eq. (20), it is fined that $\boldsymbol{v}_{z}$ $\left(=k_{1} \omega_{1}\right)$ is unchanging, which means that the essence

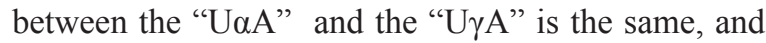
their axial movement performance is the same. Due to controlling less synchronal axis being relatively simple, the scheme of the "U $\gamma \mathrm{A}$ " is suitable to adopt.

\subsection{Analysis of Extra Rotation Performance}

Through analyzing the hobbing methods under the same profile movement (e.g. "U") and the same axial movement (e.g. $\gamma$ ), it can be concluded that they have the same precision and efficiency regardless of the extra rotation of the workpiece ("A") or extra rotation of hob (B). To the scheme "U $\gamma \mathrm{B}$ ", $\Delta \omega_{b}$ is attached to hob rotation, which causes angular acceleration of the synthesis angular velocity $\omega_{b}^{*}$ to be nonzero. The machine should adopt a servomotor driver, which would restrict its output power. However, $\Delta \omega_{b}$ is attached to workpiece rotation in the scheme "U $\gamma \mathrm{A}$ ", and there is little difference between the synthesis angular velocity $\omega_{c}^{*}$ and $\omega_{c}$, which increases no difficulty in control. In this case, the hob can be driven by an AC motor, and its angle or angular velocity can be measured by a detecting element. The testing signal can be turned into a digital signal, as a fundamental frequency, which can make up a double-fundamentalfrequency control system with another fundamental frequency that controls the $\boldsymbol{v}_{z}$ [15]. Therefore, the scheme "U $\gamma \mathrm{A}$ " is the best.

\section{HOBBING TESTS AND DETECTION FOR TOOTH FLANKS}

The excellent scheme of the "U $\gamma \mathrm{A}$ " has been brought into operation based on a hobbing platforms with ARM (Advanced RISC Machines) \& DSP (Digital Signal Processor) \& FPGA (Field Programmable Gata Array) [16]. The actual hobbing process with the elliptical helical gear hobbed is presented in Fig. 7 , from which we find that the gear can be hobbed corrected.

The surface roughness of the 1 st tooth $(\theta=$ $\left.1.3^{\circ}\right)$ and the $17^{\text {th }}$ tooth $\left(\theta=187^{\circ}\right)$ of the elliptical helical gear can be detected by a surface roughness measuring instrument [17]. The point cloud figures of roughness for tooth flanks are shown in Figs. 8a and $\mathrm{b}$, which shows that both of the two are very close, and there is no discernible difference between the two tooth flanks including their profile precision and their axial precision. Therefore, a conclusion that all of the profile precisions of the gear are uniform can be

drawn. Compared to the results between the computer simulations and the experimental studies, the results have shown that they are in good agreement.
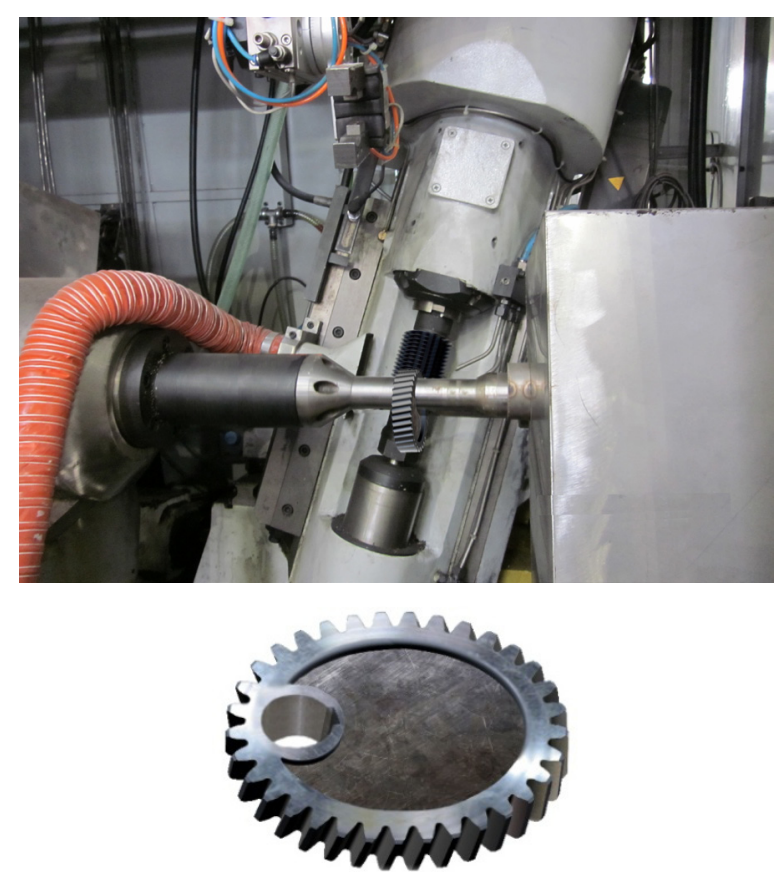

Fig. 7. Machining process and elliptic helical gear

a)

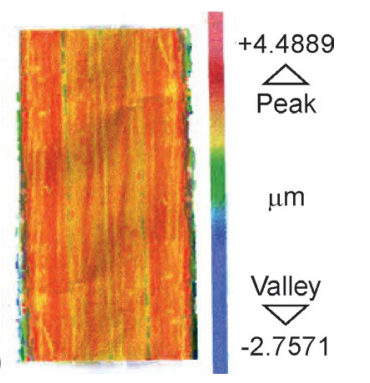

Fig. 8. Point cloud of roughness for tooth flanks, a) $\theta=187^{\circ}$, b) $\theta=1.3^{\circ}$

\section{CONCLUSIONS}

(1) The hobbing strategy based on the four-axis linkage has been built by the generating method of helical tooling rack, and some fundamental hobbing models have been deduced, which includes profile-linkage models and extra rotation models. These works make the hobbing of noncircular helical gears possible.

(2) Eighteen kinds of schemes and functional models have been developed based on the fundamental hobbing models built, according to the variety of hobbing processes in profile movement, axial 
movement, and extra rotation. Profile precision is determined only by methods of profile movement, and its axial precision is determined only by those of axial movement, and control performance is determined only by those of extra rotation. The excellent strategy and model have been selected orderly.

(3) The scheme of the "UrA" is an excellent linkage obtained, the mathematical model of which is the simultaneous Eqs. (18) and (20). Morever,

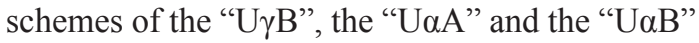
can also realize a hobbing with high precision and high efficiency, while those machine tools have a special requirement, and the control systems can also meet the requirement of dynamic characteristics. Then, those schemes are also available as options.

(4) Through the hobbing tests, it has been concluded that the excellent strategy and the model are correct and feasible. The results between the tests for tooth flanks and the computer simulations are in good agreement.

\section{ACKNOWLEDGEMENTS}

This work was financially supported by the Special Funds of the National Natural Science Foundation of China (Grant No. 51275147).

\section{REFERENCES}

[1] Litvin, F.L., Gonzalez-Perez, I., Fuentes, A. (2008). Design and investigation of gear drives with noncircular gears applied for speed variation and generation of function. Computer Methods in Applied Mechanics and Engineering, vol. 197, no. 45-48, p. 3783-3802, DOI:10.1016/j.cma.2008.03.001.

[2] Wayne, C. (2008). A new tolerance modeling and analysis methodology through a two-step linearization with applications in automotive body assembly. Journal of Manufacturing Systems, vol. 27, no. 1, p. 2635, DOI:10.1016/j.jmsy.2008.05.002.

[3] Petre, A., Dragoş, M., Cătălin, A. (2012). A gear with translational wheel for a variable transmission ratio and applications to steering box. Mechanism and Machine Theory, vol. 52, no. 6, p. 267-276, DOI:10.1016/j. mechmachtheory.2012.02.005.

[4] Mundo, D., Gatti, G., Dooner, D.B. (2009). Optimized five-bar linkages with non-circular gears for exact path generation. Mechanism and Machine Theory, vol. 44, no. 4, p. 751-760, DOI:10.1016/j. mechmachtheory.2008.04.011.

[5] Erika, O., Domenico, M., Guido, A.D., Marco, C. (2008). Numerical and experimental analysis of non-circular gears and cam-follower systems as function generators. Mechanism and Machine Theory, vol. 43, no. 8, p. 996-1008, DOI:10.1016/j. mechmachtheory.2007.07.004.

[6] Wu, C.Y., Jin, Y.Z., He, L.Y. (2008). Numerical algorithm of tooth profile of noncircular gear based on the characteristics of cutter envelope. China Mechanical Engineering, vol. 19, no. 15, p. 1796-1799. (in Chinese)

[7] Huertas Talón, J.L., Cisneros Ortega, J.C., Cisneros Ortega, L., Ros Sancho, E., Faci Olmos, E. (2010). Manufacture of a spur tooth gear in Ti-6Al-4V alloy by electrical discharge. Computer-Aided Design, vol.42, no. 3, p. 221-230, DOI:10.1016/j.cad.2009.11.001.

[8] Guan, B.G., Liao, J.H., Meng, H.Y., Liu, S.H., Chang, M. (2005). Study of cutting precision gear wheel with fiber laser. Applied Laser, vol. 25, no. 6, p. 365-368. (in Chinese)

[9] Hou, D.H., Liu, Z.M. (2003). Meshing theory analysis model for the manufacturing of helical noncircular gear by the helical tooling rack generating method. Chinese Journal of Mechanical Engineering, vol. 39, no. 8, p. 49-54, DOI:10.3901/JME.2003.08.049. (in Chinese)

[10] Hu, C.B., Ding, H.Y., Yan, K.M., Wu, Z.X. (2005). Simultaneous-control model for hobbing of noncircular helical gears. Journal of Lanzhou University of Technology, vol. 31, no. 24, p. 43-45. (in Chinese)

[11] Meredith, L.G., Radestock, M. (2005). A reflective higher-order calculus. Electronic Notes in Theoretical Computer Science, vol. 141, no. 5, p. 49-67, DOI:10.1016/j.entcs.2005.05.016.

[12] Bouzakis, K.-D., Friderikos, O., Tsiafis, I. (2008). FEM-supported simulation of chip formation and flow in gear hobbing of spur and helical gears. CIRP Journal of Manufacturing Science and Technology, vol. 1, no. 1, p. 18-26, DOI:10.1016/j.cirpj.2008.06.004.

[13] Fetvaci, C. (2010). Generation simulation of involute spur gears machined by pinion-type shaper cutters. Strojniški vestnik - Journal of Mechanical Engineering, vol. 56, no. 10, p. 644-652.

[14] Vijay Sekar, K.S., Pradeep Kumar, M. (2012). Optimising flow stress input for machining simulations using Taguchi methodology. International Journal of Simulation Modelling, vol. 11, no. 1, p. 17-28, DOI:10.2507/IJSIMM11(1)2.195.

[15] Staniek, R. (2011). Shaping of Face Toothing in Flat Spiroid Gears. Strojniški vestnik - Journal of Mechanical Engineering, vol. 57, no. 1, p. 47-54, DOI:10.5545/sv-jme.2010.093.

[16] Fei, J.Y., Deng, R., Zhang, Z., Zhou, M. (2011). Research on Embedded CNC Device Based on ARM and FPGA. Procedia Engineering, vol. 16, no. 11, p. 818-824, DOI:10.1016/j.proeng.2011.08.1160.

[17] Abouelatta, O.B., Mádl, J., Zhou, M. (2001). Surface roughness prediction based on cutting parameters and tool vibrations in turning operations. Journal of Materials Processing Technology, vol. 118, no. 1-3, p. 269-277, DOI:10.1016/S0924-0136(01)00959-1. 